\title{
SOME RESULTS ON THE SECOND BOUNDED COHOMOLOGY OF A PERFECT GROUP
}

\author{
HeE SOOK PARK
}

\begin{abstract}
For a discrete group $G$, the kernel of a homomorphism from bounded cohomology $\widehat{H}^{*}(G)$ of $G$ to the ordinary cohomology $H^{*}(G)$ of $G$ is called the singular part of $\widehat{H}^{*}(G)$. We give some results on the space of the singular part of the second bounded cohomology of $G$. Also some results on the second bounded cohomology of a uniformly perfect group are given.
\end{abstract}

\section{Introduction}

Throughout this paper, every group is considered as a discrete group.

We review the definition of bounded cohomology.

Let $G$ be a group. For a positive integer $n \geq 1$, let $C^{n}(G)$ be the space of all functions $G^{n} \rightarrow \mathbb{R}$, where $G^{n}=\underbrace{G \times G \times \cdots \times G}_{n}$. The ordinary cohomology of $G$ with real coefficients is given by the cohomology of the complex

$$
0 \stackrel{\partial_{-1}=0}{\longrightarrow} \mathbb{R} \stackrel{\partial_{0}=0}{\longrightarrow} C(G) \stackrel{\partial_{1}}{\longrightarrow} C^{2}(G) \stackrel{\partial_{2}}{\longrightarrow} C^{3}(G) \stackrel{\partial_{3}}{\longrightarrow} \cdots,
$$

where the boundary operator $\partial_{n}$ for $n \geq 1$ is defined by the formula

$$
\begin{aligned}
& \partial_{n}(f)\left(g_{1}, \cdots, g_{n+1}\right)=f\left(g_{2}, \cdots, g_{n+1}\right) \\
& +\sum_{i=1}^{n}(-1)^{i} f\left(g_{1}, \cdots, g_{i} g_{i+1}, \cdots, g_{n+1}\right)+(-1)^{n+1} f\left(g_{1}, \cdots, g_{n}\right) .
\end{aligned}
$$

We denote it by $H^{*}(G)$. Let $B^{n}(G)$ be the space of all bounded functions $G^{n} \rightarrow \mathbb{R}$, that is,

$$
B^{n}(G)=\left\{f: G^{n} \rightarrow \mathbb{R} \mid\|f\|<\infty\right\},
$$

Received June 1, 2010. Accepted June 8, 2010.

Keywords : The second bounded cohomology, perfect groups

AMS classification : Primary: 55N35; Secondary: 20J06 
where $\|f\|=\sup \left\{\left|f\left(g_{1}, \cdots, g_{n}\right)\right| \mid\left(g_{1}, \cdots, g_{n}\right) \in G^{n}\right\}$. It is easy to check that the sequence

$$
0 \rightarrow \mathbb{R} \stackrel{d_{0}=0}{\longrightarrow} B(G) \stackrel{d_{1}}{\longrightarrow} B^{2}(G) \stackrel{d_{2}}{\longrightarrow} B^{3}(G) \stackrel{d_{3}}{\longrightarrow} \cdots
$$

is a complex, where the boundary operator $d_{n}$ is defined by the same formula as in (1.1).

Definition 1.1. The $n$-th cohomology of the complex (1.2) is called the $n$-th bounded cohomology of $G$ with trivial coefficients $\mathbb{R}$ and is denoted by $\widehat{H}^{n}(G)$.

Notice that both the ordinary cohomology $H^{*}(G)$ and bounded cohomology $\widehat{H}^{*}(G)$ of a group $G$ are the vector spaces over $\mathbb{R}$. From now on, we will understand the dimension of $\widehat{H}^{n}(G)$ as its dimension as a vector space over $\mathbb{R}$.

Notice that for any group $G$, from the complex in (1.2), we have

$$
\widehat{H}^{0}(G)=\operatorname{ker}\left(d_{0}\right)=\mathbb{R} .
$$

Also

$$
\begin{aligned}
\operatorname{ker}\left(d_{1}\right) & =\left\{f \in B(G) \mid d_{1}(f)=0\right\} \\
& =\left\{f \in B(G) \mid f\left(g_{2}\right)-f\left(g_{1} g_{2}\right)+f\left(g_{1}\right)=0 \quad \text { for } g_{1}, g_{2} \in G\right\} .
\end{aligned}
$$

Thus $\operatorname{ker}\left(d_{1}\right)$ is the space of all bounded homomorphisms $G \rightarrow \mathbb{R}$. Since there is no bounded homomorphisms $G \rightarrow \mathbb{R}$, we have

$$
\widehat{H}^{1}(G)=\operatorname{ker}\left(d_{1}\right)=0 .
$$

Thus, for any group $G$ the second bounded cohomology of $G$ with trivial coefficients $\mathbb{R}$ should be investigated first. In [5] and [3] the second bounded cohomology of some interesting groups are calculated.

Amenable groups play a special role in the theory of bounded cohomology. Recall that a group $G$ is called amenable if it admits an invariant mean, that is, if there is a linear map $m: B(G) \rightarrow \mathbb{R}$ satisfying the following three conditions:

i. $m(f) \geq 0$ if $f \geq 0$

ii . $m\left(1_{G}\right)=1$, where $1_{G}$ is the constant function taking the value 1 everywhere on $G$,

iii. $m(g \cdot f)=m(f)$ for every $g \in G$ and $f \in B(G)$, where $(g \cdot f)(x)=$ $f\left(g^{-1} x\right)$.

For example, finite groups, abelian groups, solvable groups, subgroups and the homomorphic image of an amenable group are amenable. It is 
known that no group which contains a free group on two generators can be amenable.

In [4], the following is proved.

Theorem 1.1. If a group $G$ is amenable, then $\widehat{H}^{n}(G)$ is zero for every $n \geq 1$.

Thus, in particular, $\widehat{H}^{2}(G)$ is zero for an amenable group $G$. On the other hand, we have the following.

Theorem 1.2. Let $F$ be a free group of rank greater than 1. Then $\widehat{H}^{2}(F)$ is infinite dimensional as a vector space over $\mathbb{R}$.

In [3] Grigorchuk proved Theorem 1.2 by constructing explicitly the infinitely many linearly independent generators based on pseudocharacters (Definition 2.1).

From the inclusion $i_{*}: B^{*}(G) \hookrightarrow C^{*}(G)$, we consider the following commutative diagram of complexes

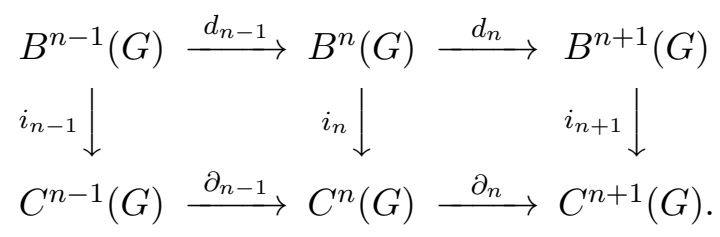

It is easy to see that $\operatorname{ker} d_{*} \subset \operatorname{ker} \partial_{*}$. Notice that the inclusion $B^{*}(G) \hookrightarrow$ $C^{*}(G)$ induces a homomorphism $\widehat{H}^{*}(G) \rightarrow H^{*}(G)$ which is in general neither injective nor surjective.

Definition 1.2. We define a vector space $\widehat{H}_{s}^{n}(G) \subset \widehat{H}^{n}(G)$ by

$$
\widehat{H}_{s}^{n}(G)=\left(I m \partial_{n-1} \cap \operatorname{ker} d_{n}\right) / I m d_{n-1}
$$

an we call $\widehat{H}_{s}^{*}(G)$ the singular part of $\widehat{H}^{*}(G)$.

Similarly, we define a vector space $H_{b}^{n}(G) \subset H^{n}(G)$ by

$$
H_{b}^{n}(G)=\operatorname{ker} d_{n} /\left(\operatorname{Im} \partial_{n-1} \cap \operatorname{ker} d_{n}\right)
$$

and we call $H_{b}^{*}(G)$ the bounded part of $H^{*}(G)$.

Notice that the spaces $\widehat{H}_{s}^{n}(G) \subset \widehat{H}^{n}(G)$ and $H_{b}^{n}(G) \subset H^{n}(G)$ are the kernel and the image, respectively, of the induced homomorphism $\widehat{H}^{*}(G) \rightarrow H^{*}(G)$.

Theorem 1.3. There is an isomorphism of vector spaces

$$
\widehat{H}^{*}(G) \cong \widehat{H}_{s}^{*}(G) \oplus H_{b}^{*}(G) .
$$


This is Corollary 1.15 in [3].

The singular part of the second bounded cohomology is more subtle and is closely related to the dimension of $\widehat{H}^{2}(G)$ for many interesting groups, such as free groups, surface groups and knot groups [3]. In the following section, we find some properties of $\widehat{H}_{s}^{2}(G)$ in terms of the pseudocharacters. We also investigate the dimension of the second bounded cohomology for a uniformly perfect group.

Acknowledgement This research was supported by Basic Science Research Program through the National Research Foundation of Korea (NRF) funded by the Ministry of Education, Science and Technology (2010-0001651).

\section{The second bounded cohomology of a perfect group}

In [3], Grigorchuck described the singular part $\widehat{H}_{s}^{2}(G)$ in terms of pseudocharacters.

Definition 2.1. A function $f: G \rightarrow \mathbb{R}$ is called a quasicharacter if there a constant $C \geq 0$ such that for every $x, y \in G$

$$
|f(x)-f(x y)+f(y)| \leq C .
$$

A quasicharacter $f: G \rightarrow \mathbb{R}$ is called a pseudocharacter if $f\left(g^{n}\right)=n \cdot f(g)$ for every integer $n$ and every $g \in G$.

We denote by $Q X(G)$ the vector space of all quasicharacters of $G$ and by $X(G)$ the space of all additive characters (homomorphisms) $f: G \rightarrow$ $\mathbb{R}$. We also denote by $P X(G)$ the space of all pseudocharacters of $G$.

Theorem 2.1. There is an isomorphism of vector space

$$
\widehat{H}_{s}^{2}(G) \cong P X(G) / X(G) .
$$

This is Theorem 3.5 in [3].

Proposition 2.2. If $\widehat{H}_{s}^{2}(G) \neq 0$, then there exists at least one element $x \in G$ of infinite order.

Proof. Since $\widehat{H}_{s}^{2}(G) \neq 0$, there is at least one pseudocharacter $\alpha \in$ $P X(G) \backslash X(G)$. Then $\alpha\left(g^{n}\right)=n \cdot \alpha(g)$ for every integer $n$ and $g \in G$. This shows that $\alpha(g)=0$ for every $g \in G$ of finite order. Since $\alpha \neq 0$, there must be at least one element $x \in G$ such that $\alpha(x) \neq 0$ and so the order of $x$ is infinite. 
Corollary 2.3. Let $G$ be a torsion group. Then the dimension of $\widehat{H}^{2}(G)$ is less than or equal to the dimension of $H^{2}(G)$.

Proof. Since $G$ is a torsion group, the order of every element of $G$ is finite. Hence by Proposition 2.2, every pseudocharacter of $G$ is zero and the singular part of the second bounded cohomology of $G$ is zero. Thus the induced homomorphism $\widehat{H}^{2}(G) \rightarrow H^{2}(G)$ is injective.

Corollary 2.4. Let $\widehat{H}_{s}^{2}(G) \neq 0$. Then $G$ contains an infinite cyclic group.

Proof. From Proposition 2.2, $G$ contains an element $x$ of infinite order. The group generated by $x$ is an infinite cyclic subgroup of $G$.

Remark 2.1. Let $G=\mathbb{Z} * \mathbb{Z}$ a free group of rank 2 , generated by $x$ and $y$. First recall that the ordinary cohomology of a free group is zero for every degree greater than 1 . Hence $H^{2}(G)=0$. On the other hand, by Theorem 1.2, $\widehat{H}^{2}(G)$ is infinite dimensional. Hence $\widehat{H}^{2}(G)=\widehat{H}_{s}^{2}(G)$. In [3], it is proved that

$$
\widehat{H}_{s}^{2}(G) \cong P X_{0}(G),
$$

where $P X_{0}(G)$ is a space of pseudochatacters of $G$ vanishing on the generators $x$ and $y$.

Recall that there is a five-term exact sequence in the ordinary cohomology [2]:

Theorem 2.5. Let $N \unlhd G$ be a normal subgroup of $G$. Then there is an exact sequence

$$
0 \rightarrow H^{1}(G / N) \rightarrow H^{1}(G) \rightarrow H^{1}(N)^{G / N} \rightarrow H^{2}(G / N) \rightarrow H^{2}(G),
$$

where $H^{1}(N)^{G / N}$ is a $G / N$-invariant subspace of $H^{*}(N)$.

Similarly, there is a five-term exact sequence in the bounded cohomology:

Theorem 2.6. Let $N \unlhd G$ be a normal subgroup of $G$. Then there is an exact sequence

$$
0 \rightarrow \widehat{H}^{2}(G / N) \rightarrow \widehat{H}^{2}(G) \rightarrow \widehat{H}^{2}(N)^{G / N} \rightarrow \widehat{H}^{3}(G / N) \rightarrow \widehat{H}^{3}(G),
$$

where $\widehat{H}^{2}(N)^{G / N}$ is a $G / N$-invariant subspace of $\widehat{H}^{2}(N)$.

Proof. This is Theorem 12.4.2 in [6] 
Proposition 2.7. Let $G=\langle x\rangle *\langle y\rangle$ a free group generated by $x$ and $y$. Let $N$ be the normal closure of $\langle y\rangle$. Then $N$ is a proper normal subgroup of $G$ and infinitely generated. Furthermore, $\widehat{H}^{2}(N)$ is infinite dimensional.

Proof. By definition, it is clear that $N$ is a nontrivial normal subgroup of $G$. As it is well known, we have $G / N \cong<x>$. Hence the index of $N$ in $G$ is infinite and $\langle y\rangle \subset N \neq G$. Also recall that a finitely generated normal subgroup of a nontrivial free group $F$ is of finite index in $F$. Hence $N$ must be infinitely generated. Since $G / N \cong\langle x\rangle$, the quotient $G / N$ is abelian and so amenable. So by Theorem 1.1, $\widehat{H}^{n}(G / N)=0$ for every $n \geq 1$. Hence by Theorem 2.6 there is an isomorphism of vector spaces

$$
\widehat{H}^{2}(G) \cong \widehat{H}^{2}(N)^{G / N} .
$$

Since $\widehat{H}^{2}(G)$ is infinite dimensional by Theorem $1.2, \widehat{H}^{2}(N)^{G / N}$ is infinite dimensional and so is $\widehat{H}^{2}(N)$.

Notice that for a normal subgroup $N \unlhd G$, if the quotient $G / N$ is amenable, then there the inclusion homomorphism $N \hookrightarrow G$ induces an injective homomorphism $\widehat{H}^{2}(G) \rightarrow \widehat{H}^{2}(N)$. For example, for the commutator subgroup $[G, G]$ of $G$, the quotient $G /[G, G]$ is abelian and so amenable. Thus there is an injective homomorphism $\widehat{H}^{2}(G) \rightarrow$ $\widehat{H}^{2}([G, G])$.

As a corollary to Theorem 2.6, we prove that Lyndon-HochschildSerre spectral sequence for $\widehat{H}^{2}(G)$.

Corollary 2.8. Let $N$ be a normal subgroup of $G$. Suppose $\widehat{H}^{2}(G / N)$ $=0$. Then there the spectral sequence $E_{2}^{p q}=\widehat{H}^{p}\left(G / N, \widehat{H}^{q}(N)\right)$ converging to $\widehat{H}^{2}(G)$.

Proof. Suppose $\widehat{H}^{2}(G / N)$ is zero. Then by Theorem 2.6 the homomorphism $\widehat{H}^{2}(G) \rightarrow \widehat{H}^{2}(N)^{G / N}$ is injective. For

$$
E_{2}^{p q}=\widehat{H}^{p}\left(G / N, \widehat{H}^{q}(N)\right),
$$

we fix $(p, q)=(0,2)$. Then we have

$$
E_{2}^{0,2}=\widehat{H}^{0}\left(G / N, \widehat{H}^{2}(N)\right)=\widehat{H}^{2}(N)^{G / N} .
$$

Consider

$$
0=E_{2}^{-2,3} \rightarrow E_{2}^{0,2}=\widehat{H}^{2}(N)^{G / N} \stackrel{d_{2}}{\longrightarrow} E_{2}^{2,1}=\widehat{H}^{2}\left(G / N, \widehat{H}^{1}(N)\right)=0,
$$


where the last term is zero because the first bounded cohomology of any group is zero. This gives

$$
E_{3}^{0,2}=\operatorname{ker} d_{2}=E_{2}^{0,2}=\widehat{H}^{2}(N)^{G / N} .
$$

For $E_{4}^{0,2}$, we consider

$0=E_{3}^{-3,4} \rightarrow E_{3}^{0,2}=\widehat{H}^{2}(N)^{G / N} \stackrel{d_{3}}{\longrightarrow} E_{3}^{3,0}=\widehat{H}^{3}\left(G / N, \widehat{H}^{0}(N)\right)=\widehat{H}^{3}(G / N)$,

where the last term follows from $\widehat{H}^{0}(N)=\mathbf{R}$. Then by the exactness of the five-term exact sequence above, we have

$$
\begin{aligned}
E_{4}^{0,2} & =\operatorname{ker} d_{3}=\operatorname{ker}\left(\widehat{H}^{2}(N)^{G / N} \rightarrow \widehat{H}^{3}(G / N)\right) \\
& =\text { image of }\left(\widehat{H}^{2}(G) \hookrightarrow \widehat{H}^{2}(N)^{G / N}\right) \\
& \cong \widehat{H}^{2}(G) .
\end{aligned}
$$

Thus

For $r=4$, we consider

$$
E_{4}^{0,2} \cong \widehat{H}^{2}(G)
$$

$$
0=E_{4}^{-4,5} \stackrel{d_{4}}{\longrightarrow} E_{4}^{0,2}=\widehat{H}^{2}(G) \stackrel{d_{4}}{\longrightarrow} E_{4}^{4,-1}=0 .
$$

So $E_{5}^{0,2}=\operatorname{ker} d_{4}=E_{4}^{0,2}$. Now it is easy to see that for every $r \geq 5$ we have

$$
E_{r}^{0,2}=E_{4}^{0,2}=\widehat{H}^{2}(G) .
$$

Hence we have $E_{r}^{0,2}$ converges to $\widehat{H}^{2}(G)$.

Corollary 2.8 is still true without the condition $\widehat{H}^{2}(G / N)=0$. Related to Theorem 2.6, the following more general version of LyndonHochschild-Serre spectral sequence is proved in [7]:

Lemma 2.9. Let $N$ be a normal subgroup $N \unlhd G$. Then $\widehat{H}^{*}(G / N)$ admits a natural structure of bounded $G / N$-module and there exists a spectral sequence $\left(E_{r}\right)$ converging to $\widehat{H}^{*}(G)$ in which $E_{2}^{p q}=\widehat{H}^{p}\left(G / N, \widehat{H}^{q}\right.$ $(N))$ for every $p, q \geq 0$.

Recall that a subgroup of a free group is also free. Proposition 2.7 gives another example for the fact that the rank of a subgroup of a free group of finite rank is not necessarily finite.

Recall that we say a group $G$ is perfect if $G=[G, G]$, where $[G, G]$ is the commutator subgroup of $G$. Recall that $H_{1}(G, \mathbb{Z}) \cong G /[G, G]$. Hence $H^{1}(G)=X(G)=0$ for a perfect group $G$. Thus from Theorem 2.1, we have $\widehat{H}_{s}^{2}(G) \cong P X(G)$. 
Definition 2.2. A group $G$ is said to be uniformly perfect if there is a positive integer $N$ such that every element of $G$ can be presented as a product of at most $N$ commutators.

For example, it is known that the alternating groups $A_{n}$ for $n \geq 5$ and $G=S L(2, \mathbb{R})$ are uniformly perfect $[8]$.

In [5], the following theorem is proved:

Theorem 2.10. If $G$ is uniformly perfect, then homomorphism $\widehat{H}^{2}(G)$ $\rightarrow H^{2}(G)$ is injective.

Definition 2.3. A group such that $H_{1}(G, \mathbb{Z})=H_{2}(G, \mathbb{Z})=0$ is called superperfect. Furthermore, if $H_{n}(G, \mathbb{Z})=0$ for all $n \geq 1$, then $G$ is called acyclic.

Notice that acyclic groups are superperfect, and superperfect groups are perfect. The alternating group $A_{5}$ is (uniformly) perfect, but not superperfect. If $G$ is superperfect, then by Universal Coefficient Theorem $H^{1}(G)=H^{2}(G)=0$. Also, since $H^{1}(X)=X(G)$, we have

$$
\widehat{H}^{2}(G)=\widehat{H}_{s}^{2}(G) \cong P X(G) / X(G)=P X(G) .
$$

One of interesting examples of acyclic groups is binate groups [1].

Definition 2.4. A group $G$ is called binate if it is the direct limit of subgroups $G_{\lambda}$ where for each $\lambda$ there exist $\mu \geq \lambda, u_{\lambda} \in G_{\mu}-G_{\lambda}$ and $\phi_{\lambda}: G_{\lambda} \rightarrow G_{\mu}$ such that $g=\left[u_{\lambda}, \phi_{\lambda} g\right]$ for all $g \in G_{\lambda}$.

As shown in [1], a binate group $G$ is acyclic and so superperfect. By Universal Coefficient Theorem, $H^{n}(G)=0$ for every $n \geq 1$. In particular, $H^{1}(G)=H^{2}(G)=0$. Also it is shown in [1] that every element of a binate group $G$ is a commutator. Thus it is uniformly perfect. Hence $\widehat{H}^{2}(G)=0$ by Theorem 2.10. Then it is clear that $P X(G)=0$.

Recall that the quotient of a perfect group is also perfect.

Proposition 2.11. Let $Z$ be the center of a superperfect group $G$. If the quotient $G / Z$ is uniformly perfect and $\widehat{H}^{2}(G) \neq 0$, then the center $Z$ is nontrivial. In particular, $Z$ has at least one element of infinite order.

Proof. By Theorem 2.6, the exact sequence $0 \rightarrow Z \rightarrow G \rightarrow G / Z \rightarrow 1$ induces a five-term exact sequence

$$
0 \rightarrow \widehat{H}^{2}(G / Z) \rightarrow \widehat{H}^{2}(G) \rightarrow \widehat{H}^{2}(Z) \rightarrow \widehat{H}^{3}(G / Z) \rightarrow \widehat{H}^{3}(G) .
$$

First notice that, since $Z$ is the center of $\mathrm{G}$, the conjugation action of $G / Z$ on $\widehat{H}^{2}(Z)$ is trivial and so $\widehat{H}^{2}(Z)^{G / Z} \cong \widehat{H}^{2}(Z)$. Recall that the 
center $Z$ is abelian and so it is amenable. So by Theorem $1.1 \widehat{H}^{*}(Z)=0$. Hence there is an isomorphism

$$
\widehat{H}^{2}(G / Z) \cong \widehat{H}^{2}(G)
$$

and so $\widehat{H}^{2}(G / Z) \neq 0$. Since $G / Z$ is uniformly perfect, there is an injective homomorphism $\widehat{H}^{2}(G / Z) \hookrightarrow H^{2}(G / Z)$ by Theorem 2.10 and so $H^{2}(G / Z) \neq 0$. Recall that $G$ is perfect. So the quotient $G / Z$ is also perfect and $H^{1}(G / Z)=0$. Also notice that $H^{2}(G)=0$. Thus from Theorem 2.5, there is an isomorphism $H^{1}(Z) \cong H^{2}(G / Z)$. Hence $H^{1}(Z) \neq 0$ so that $Z$ is nontrivial. If every element of $Z$ is torsion, then $0=X(Z) \cong H^{1}(Z)$. Hence there is at least one element $z \in Z$ such that the order of $z$ is infinite.

Remark 2.2. From the Proposition 2.11, notice that

$$
\widehat{H}^{2}(G / Z) \cong \widehat{H}^{2}(G)=\widehat{H}_{s}^{2}(G)
$$

and $\widehat{H}_{s}^{2}(G) \neq 0$. However, $\widehat{H}_{s}^{2}(G / Z)=0$. Thus, even the spaces of the second bounded cohomology of two groups are isomorphic, their singular parts are not necessarily isomorphic.

Definition 2.5. A group $G$ is of type $F P_{n}$ if $\mathbb{Z}$ is type of $F P_{n}$ as a $\mathbb{Z} G$-module. More precisely, there is a partial projective resolution $P_{n} \rightarrow \cdots \rightarrow P_{0} \rightarrow \mathbb{Z} \rightarrow 0$ of finite type, that is, such that each $P_{k}$ is finitely generated for $0 \leq k \leq n$.

As proved in [2], a group $G$ is of type $F P_{n}$ if and only if for every partial projective resolution $P_{k} \rightarrow \cdots P_{0} \rightarrow \mathbb{Z} \rightarrow 0$ of finite type with $k<n, \operatorname{ker}\left\{P_{k} \rightarrow P_{k-1}\right\}$ is finitely generated.

Proposition 2.12. If a group $G$ is of type $F P_{n}$, then the dimension of $H^{k}(G)$ is finite for $k \leq n$.

Proof. Since $G$ is of type $F P_{n}$ and $\mathbb{Z}$ is finitely generated abelian group, every $H_{k}(G, \mathbb{Z})$ for $k \leq n$ is a finitely generated abelian group. Hence we can write

$$
H_{k}(G, \mathbb{Z}) \cong \mathbb{Z}^{r_{k}} \oplus T_{k},
$$

where the rank $r_{k} \geq 0$ is finite and $T_{k}$ is the torsion subgroup of $H_{k}(G, \mathbb{Z})$. Then

$$
H^{k}(G) \cong \operatorname{Hom}_{\mathbb{Z}}\left(H_{k}(G, \mathbb{Z}), \mathbb{R}\right)=\mathbb{R}^{r_{k}},
$$

where the first isomorphism follows from Universal Coefficient Theorem. Hence the dimension of $H^{k}(G)$ is finite. 
Remark 2.3. Recall that the group $H_{2}(G, \mathbf{Z})$ is called the Schur multiplier of $G$. It is an important invariant of a group that has applications in many areas. Notice that a superperfect group is one whose abelianization and also Schur multiplier vanish. A group $G=F / K$ with $F$ free, it is known [2] that $H_{2}(G, \mathbb{Z}) \cong(K \cap[F, F]) /[F, K]$.

We first see how the dimension of the second bounded cohomology of a uniformly perfect group $G$ which is of type $F P_{2}$ is affiliated with $H_{2}(G, \mathbb{Z})$.

Corollary 2.13. Let $G$ be uniformly perfect and of type $F P_{2}$. Then the dimension of $\widehat{H}^{2}(G)$ is finite. Especially, its maximum is achieved by the rank of $H_{2}(G, \mathbb{Z})$.

Proof. Since $G$ is of type $F P_{2}$, by Proposition 2.12 the dimension of $H^{2}(G)$ is equal to the rank of $H_{2}(G, \mathbb{Z})$, which is finite. Since $G$ is uniformly perfect, there is an injective homomorphism $\widehat{H}^{2}(G) \hookrightarrow H^{2}(G)$ and so the dimension of $\widehat{H}^{2}(G)$ is less than equal to the dimension of $H^{2}(G)$.

Theorem 2.14. Let $G$ be uniformly perfect. If $G$ is finitely presented with $m$ generators and $n$ relations, then the dimension of $\widehat{H}^{2}(G)$ is at most $n-m$.

Proof. Notice that a finitely generated group is of type $\mathrm{FP}_{2}$, and so by Corollary 2.13 the dimension of $\widehat{H}^{2}(G)$ is finite. Let $r=r k_{Z}\left(G_{a b}\right)$ the rank of the abelianization of $G$. As it is well known, the group $H_{2}(G, \mathbf{Z})$ can be generated by $n-m+r$ elements. Since $G$ is uniformly perfect, it is perfect and so $r=0$. Thus $H_{2}(G, \mathbf{Z})$ can be generated by $n-m$ elements. Since $G$ is uniformly perfect, similarly as shown in Corollary 2.13 , the dimension of $\widehat{H}^{2}(G)$ is at most $n-m$.

\section{References}

[1] A topologist's view of perfect and acyclic groups, Invitations to geometry and topology, Oxf. Grad. Texts Math., 7, Oxford Univ. Press, Oxford, 2002, 1-28.

[2] K. Brown, Cohomology of Groups, Springer-Verlag, 1994

[3] R. Grigorchuk, Some results on bounded cohomology, LMS, 202, 1993, 111-163.

[4] N. Ivanov, Foundation of theory of bounded cohomology, Journal of Soviet Math., 37, 1987, 1090-1114.

[5] S. Matsumoto, S. Morita, Bounded cohomology of certain groups of homomorphisms, Proc. Amer. Math. Soc., 94, 1985, 539-544.

[6] N. Monod, Continuous bounded cohomology of locally compact groups, Lecture Notes in Masthematics 1758, Springer, 2001. 
[7] G. Noskov, The Hochschild-Serre spectral sequence for bounded cohomology, Contemp. Math. 131, 1992, 613-629.

[8] L.N. Vaserstein, Commutators in Linear groups, K-Theory 2, 761-767, 1989.

\author{
ASARC \\ KAIST \\ DaeJeon, 305-751 Republic of KOREA \\ E-mail: hseapark@kaist.ac.kr
}

